\title{
REVISIÓN SOBRE LA APLICACIÓN DE LA REALIDAD VIRTUAL EN LA REHABILITACIÓN VESTIBULAR
}

\section{Review on the application of virtual reality to vestibular rehabilitation}

\author{
Rafael ÁLVAREZ-OTERO \\ SACYL. Complejo Asistencial Universitario de Palencia. Servicio de Otorrinolaringología. Palencia. España \\ Correspondencia: dralvarez.orl@gmail.com
}

Fecha de recepción: 27 de julio de 2019

Fecha de aceptación: 8 de agosto de 2019

Fecha de publicación: 10 de agosto de 2019

Fecha de publicación del fascículo: 1 de marzo de 2020

Conflicto de intereses: Los autores declaran no tener conflictos de intereses

Figuras: Los autores declaran disponer del permiso, con atribución, para modificar y compartir las fuentes utlizadas para su elaboración.

Política de derechos y autoarchivo: se permite el autoarchivo de la versión post-print (SHERPA/RoMEO)

Licencia CC BY-NC-ND. Licencia Creative Commons Atribución-NoComercial-SinDerivar 4.0 Internacional

Universidad de Salamanca. Su comercialización está sujeta al permiso del editor

RESUMEN: Introducción y objetivo: Las nuevas tecnologías nos conceden nuevas fórmulas para analizar y modificar la relación del sujeto con el medio. La realidad virtual sería su máxima expresión. Explicaremos los fundamentos básicos que se aplican en estas tecnologías, las cuales poseen muchos elementos en común con la organización de nuestro sentido del equilibrio.

Método: Revisión narrativa. Resultados: Expondremos las características técnicas y los mecanismos de compensación aplicados en los dispositivos de rehabilitación vestibular. Discusión: Revisaremos algunos estudios que analizan las ventajas e inconvenientes de la realidad virtual frente a la terapia física convencional. Conclusiones: Los sistemas de seguimiento ocular, postural o de giro cefálico aplicados a la realidad virtual, pueden emplearse en el desarrollo de dispositivos diagnósticos o de rehabilitación vestibular. Se necesitas más estudios que comparen su eficacia respecto a la terapia física tradicional.

PALABRAS CLAVE: realidad virtual; movimientos oculares; movimientos cefálicos; pruebas vestibulares; trastornos vestibulares. 
SUMMARY: Introduction and objective: New technologies grant us new formulas to analyze and modify the subject's relationship with the environment. Virtual reality would be its maximum expression. We will explain the basic foundations that are applied in these technologies, which have many elements in common with the organization of our sense of balance. Method: Narrative review. Results: We will present the technical characteristics and compensation mechanisms applied in vestibular rehabilitation devices. Discussion: We will review some studies that analyze the advantages and disadvantages of virtual reality compared to conventional physical therapy. Conclusions: The ocular, postural or head turn monitoring systems applied to virtual reality can be used in the development of diagnostic or vestibular rehabilitation devices. More studies are needed that compare their effectiveness with respect to traditional physical therapy.

KEYWORDS: virtual reality; eye movements; head movements; vestibular function tests; vestibular diseases

\section{INTRODUCCIÓN $[1,2]$}

Las nuevas tecnologías que posibilitan una experiencia virtual inmersiva se basan en sistemas cuyo desarrollo se inicia en los años 60 en laboratorios de investigación básica, siendo hoy en día omnipresentes. Los principios técnicos de las imágenes tridimensionales $(3 \mathrm{D})$ generadas por ordenador se describen a principios de los 70 y desde entonces, el incremento en potencia de los microprocesadores ha permitido renderizar (generar una imagen realista desde un modelo 3D) entornos gráficos tridimensionales en consolas y ordenadores.

Con películas como El cortador de césped (Brett Leonard, 1992), Matrix (Wachowski, 1999) Ready Player One (Spielberg, 2018), la realidad virtual ha pasado del cine a nuestros hogares, primero como una experiencia de ciencia ficción hasta convertirse en algo tangible e incluso de moda. Avatar (Cameron, 2009) supuso el impulso para la industria cinematográfica con gafas $3 \mathrm{D}$, después del fracaso del sistema anaglifo (imágenes de dos dimensiones capaces de provocar un efecto tridimensional cuando se ven con lentes especiales (lentes de color diferente para cada ojo)). En los últimos años, casi todas las grandes empresas tecnológicas están lanzando productos relacionados con la realidad virtual o realidad aumentada.
Las especificaciones y sus dispositivos de entrada están en constante evolución, siendo muy difícil seguir su progreso.

Al mismo tiempo y de forma paralela, estas ciencias se han ido aplicando al campo de la rehabilitación vestibular, mediante la adaptación de los medios tecnológicos que nos ofrece la industria de los videojuegos o a través del desarrollo de nuevos dispositivos con una inmersión en entornos virtuales 3D más complejos.

Al producirse una lesión vestibular, se modifica la influencia relativa de los sistemas visual, vestibular y propioceptivo y mediante la rehabilitación se pretende recalibrarlo, utilizando los mecanismos de adaptación, habituación y sustitución. El empleo de nuevas herramientas aplicando estos mismos principios, servirá para valorar su capacidad diagnóstica y eficacia terapéutica.

En este artículo sintetizaremos los fundamentos básicos que se aplican en esta tecnología, utilizando diferentes combinaciones de sistemas de seguimiento ocular, postural o de giro cefálico, propios de la organización de nuestro sentido del equilibrio. Describiremos las características de estos dispositivos y su aplicación en la rehabilitación vestibular, para terminar, discutiendo las ventajas e inconvenientes de la realidad virtual frente a la terapia física convencional. 


\section{PRINCIPIOS TÉCNICOS DE LA REALIDAD VIRTUAL [2-5]}

El equilibrio corporal es el resultado de la interacción a nivel del sistema nervioso central de la información procedente de los canales sensoriales visual, vestibular y propioceptivo. De manera similar, los modelos de seguimiento o tracking de realidad virtual se basan en la integración de lo que vemos, de nuestra inclinación y del desplazamiento por el espacio, que será procesado en un ordenador o un clúster de computadoras para generar un entorno virtual. Por lo tanto, haremos un paralelismo entre estos sistemas de seguimiento y nuestros órganos de los sentidos, destacando aquellas tecnologías que forman parte de los dispositivos de uso en el medio hospitalario.

\section{SISTEMA VISUAL}

La visión binocular fisiológica tiene un ángulo aproximado de $120^{\circ}$, que añadiendo la visión periférica llegaría a $200-220^{\circ} \sin$ mover los ojos y hasta $270^{\circ}$ en mirada extrema.

- Si miramos por un sólo ojo, podemos descubrir señales que nos permiten estimar a qué distancia se encuentran las cosas: sombras, la reflexión de la luz, su tamaño relativo, su situación respecto al horizonte o a una perspectiva lineal, etc. También la diferente velocidad a la que se mueven estos objetos respecto a nosotros (efecto parallax) y la acomodación ocular, ofrecen una sensación de relieve a la visión monocular.

- La mayor parte de estos distintivos se han ido integrando en los motores que generan los entornos gráficos tridimensionales y a medida que se consigue que estos efectos se vuelvan más realistas, se necesitan computadoras más potentes para poderlos producir.

- Cuando empleamos la visión binocular o estereoscópica, nuestro sistema nervioso integra las dos imágenes ligeramente diferentes que llegan a nuestras retinas, lo que nos faculta para percibir el relieve, la profundidad o la tridimensionalidad. Se estima que entre un 5 a un $10 \%$ de la población no tiene una adecuada visión estereoscópica. Un sencillo examen oftalmológico permitiría detectar a estas personas que no pueden disfrutar correctamente de una película en 3D o de unas gafas de realidad virtual.

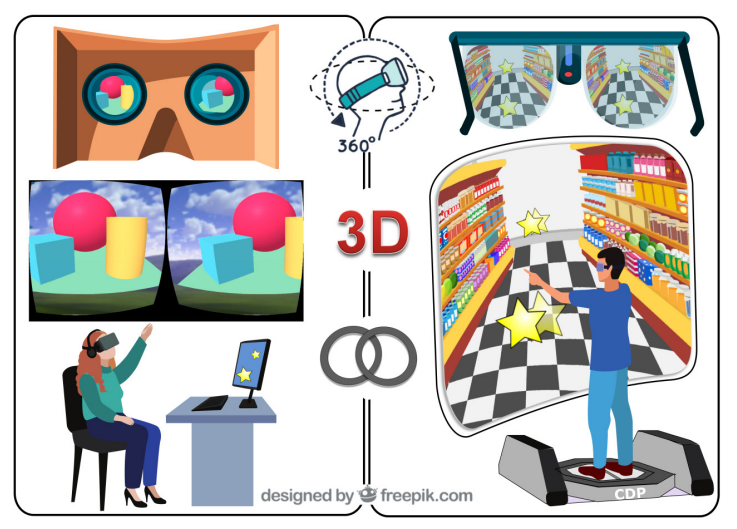

Figura 1. Izquierda: Gafas de realidad virtual tipo Google Cardboard. Se puede aprecias la diferencia entre la imagen de ojo derecho e izquierdo. Los pacientes pueden realizar ejercicios en casa aprovechando su teléfono inteligente dotado de giroscopios y de sonido estereofónico. Derecha: Dispositivo avanzado de rehabilitación vestibular que combina una plataforma de posturografía dinámica computarizada (CDP) con una pantalla donde se proyectan imágenes de realidad virtual que se visualizan en tres dimensiones (3D) con gafas con filtro de luz polarizada. Puede emplearse con fines diagnósticos y terapéuticos.

Imagen creada a partir de http://www.freepik.com

Hace años que llevan aplicándose diferentes técnicas de visión estereoscópica a la industria del ocio, entre ellas:

- Gafas anaglifos (las clásicas con un cristal rojo y otro cían).

- Estéreo pasivo (passive stereo), (Figura 1, derecha) precisan monitores o proyectores que emplean filtros de polarización activos o dos emisiones con diferentes filtros. Mediante gafas de cristales polarizados que tienen 
lentes con el filtro adecuado para cada ojo, se separan las dos emisiones.

- Estéreo activo (active stereo): alternativamente un cristal de las gafas se opacifica de manera sincronizada con el proyector o monitor para mostrar diferentes imágenes en cada ojo.

- Autoestéreo: presente, por ejemplo, en la consola Nintendo 3DS. Emplea una máscara barrera sobre la pantalla que permite presentar diferentes pixeles y por lo tanto imágenes a cada ojo.

- Lenticular lens mask realiza un efecto similar al autoestéreo, utilizando un cristal con cientos de microlentes sobre distintos grupos de píxeles.

- Gafas de realidad virtual (head mounted display o $H M D$ ) que nos muestran dos imágenes con distinto punto de perspectiva para cada ojo, de manera que al integrarlas se produce la sensación de profundidad (Figura 1, izquierda). Su principio es el mismo que el de los visores estereoscópicos de hace décadas, que exponían una imagen estática a cada ojo, sólo que ahora cada pantalla o mitad de pantalla nos muestra un video. Las gafas de realidad virtual más modernas pueden dar un ángulo de visión de 115-120 mientras que unas gafas tipo Google Cardboard sólo permiten unos $90^{\circ}$.

- Las herramientas que generan estos entornos tridimensionales aprovechan tecnologías accesibles como OpenGL o Blender, cuya limitación es la capacidad de procesamiento de los dispositivos. También podemos desarrollar juegos o aplicaciones aprovechando motores gráficos como Unity o Unreal Engine, aunque si se obtuvieran beneficios, las empresas tendrían derecho a una parte de los mismos. En el entorno web, las especificaciones de WebGL 2.0 implementadas sobre JavaScript permiten la renderización de gráficos en $3 \mathrm{D}$ en cualquier navegador moderno de nuestros teléfonos inteligentes, que no dejan de ser pequeños ordenadores.

- Entornos de inmersión como CAVE (Cave Automatic Virtual Environment) crean un hábitat virtual cúbico de hasta 3 metros de lado con retroproyectores estereoscópicos, logrando $270^{\circ}$ de visión. CAVE2 $2^{T M}[6]$ perfecciona este concepto y recrea un entorno aún más inmersivo de casi $360^{\circ}$, apoyado en múltiples pantallas planas que en conjunto dan imágenes 3D y sonido envolvente (Figura 2).

Sistemas DE SEGUIMIENTO OCULAR (eye tracking): muy similares a los desarrollados hace años para videonistagmografía o los más recientes para el video head impulse test (vHIT). Están formados por una o dos cámaras con visión infrarroja que, combinadas con un ordenador, monitorizan la posición de la pupila. En el entorno de la realidad virtual estos sistemas ya están muy avanzados y recientemente se ha lanzado al mercado un HMD de HTC, la VIVE Pro Eye. Este dispositivo tiene eye tracking y lo que se denomina foveated rendering, que luego explicaremos.

\section{SISTEMA VESTIBULAR}

El giroscopio sería al sistema vestibular el equivalente de los canales semicirculares, y el acelerómetro electrónico se correspondería con el utrículo y el sáculo. Por lo tanto, tres giroscopios medirían la inclinación o movimiento angular en los tres planos del espacio y por su parte, los acelerómetros, cuantificarían el momento o aceleración lineal.

- Los giroscopios forman parte de los instrumentos de vuelo, e incluso los encontramos en los coches todo terreno. Ahora están integrados en muchos de los teléfonos móviles de gama media alta, así como en mandos de consola. Al tener poca latencia son perfectos para cambiar la perspectiva de los visores de realidad virtual cuando giramos la cabeza. Por contra, otros sistemas de posicionamiento en el espacio precisan mayor fuerza 
de computación y por lo tanto tienen mayor latencia, lo cual genera un conflicto en la experiencia virtual.

- Los acelerómetros sirven de apoyo al giroscopio, pero en un entorno amplio de realidad virtual sólo pueden intuir algunos cambios de altura de la cabeza.

Al monitorizar el movimiento rotacional de la cabeza en los tres planos de un espacio virtual, se habla de que el dispositivo dispone de «tres grados de libertad». Estos se consiguen incluso con unas gafas de realidad virtual sencillas, como las de Google Cardboard, en la que se coloca en su interior un teléfono con giroscopio.

En la práctica clínica, un vHIT combina una cámara de alta velocidad para el seguimiento ocular con un giroscopio que cuantifica los movimientos cefálicos de rotación, con tres grados de libertad.

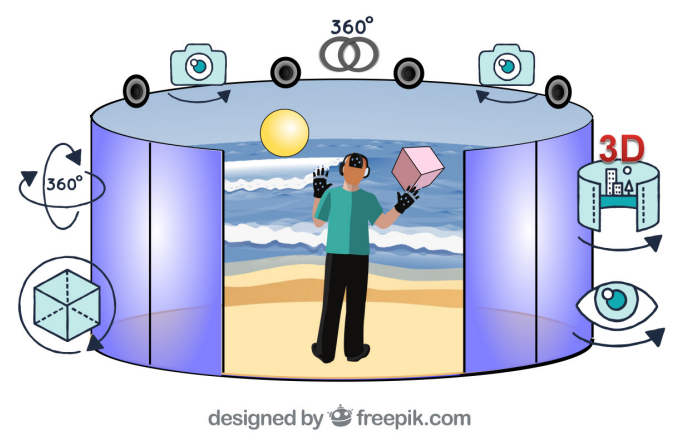

Figura 2. Ejemplo de sistema complejo de realidad virtual. Experiencia virtual con sonido envolvente e imágenes tridimensionales panorámicas. Se realiza seguimiento de la localización del sujeto y de la orientación de su cabeza en el espacio (6 grados de libertad) mediante diez cámaras de infrarrojos que triangulan la posición. La orientación la proporcionan dispositivos con luces en constelación sobre la persona. Las gafas dispondrían de cámara/s para monitorizar la posición del ojo. Imagen creada a partir de http://www.freepik.com

\section{SISTEMA PROPIOCEPTIVO}

Los sistemas de seguimiento o tracking del desplazamiento del sujeto por el espacio real permiten trasladarle a través del entorno virtual. Diferentes tecnologías con sistemas de emisor/receptor, pueden reubicar no sólo su posición sino también la disposición de sus extremidades, especialmente de las manos; pero como en el caso de la rehabilitación vestibular, la interacción de las manos con el medio es poco importante, no expondremos este tipo de tecnología, centrándonos específicamente en los diferentes sistemas de seguimiento:

- Seguimiento mecánico: se puede llevar a cabo a través de dispositivos similares a un brazo articulado, que monitorizan la posición de distintos elementos, como la cabeza o el cuerpo. Son aparatosos y por lo tanto limitan el espacio de actuación. Un ejemplo en otoneurología serían las plataformas de posturografía, cuyos transductores de fuerza localizan el centro de presiones. El seguimiento de la proyección del centro de equilibrio sobre la plataforma podría considerarse un seguimiento mecánico.

- Seguimiento electromagnético: permite medir distancia y orientación mediante emisores y una base receptora. No necesita mantener una línea de visión con el receptor, presenta poca latencia, pero está limitado por una distancia de unos tres metros. Un ejemplo de neuronavegación electromagnética es el sistema BrainLab disponible en algunos quirófanos.

- Seguimiento acústico: la emisión de ultrasonidos permite localizar objetos en el espacio. No es una tecnología novedosa pues se basa en la que permitió el sonar o la ecografía. Ofrece mayor distancia, pero tiene muchas limitaciones debido al medio o a la velocidad del sonido.

- Seguimiento óptico: los más conocidos son los sistemas de captura de movimiento, o motion 
capture, que se emplean en la industria cinematográfica para animar con movimiento real, a partir del movimiento de actores, los personajes generados por ordenador. También se utiliza para el estudio de la marcha humana con fines médicos y deportivos. Precisa dos cámaras y unos marcadores colocados en las articulaciones y partes principales del cuerpo. Estos puntos de contraste, al situarse sobre un puntero tipo varita o sobre un casco de realidad virtual, se disponen en forma de constelación para permitir el seguimiento, no sólo de la posición, sino de la orientación. Muchos de estos marcadores son pequeñas luces tipo LED infrarrojas, como en las gafas Oculus Rift, que dispone de 20 luces conformando un patrón sobre HMD, mientras dos cámaras de visión infrarroja, colocadas en dos puntos distantes de la habitación, realizan el seguimiento. Todos estos dispositivos necesitan una precisa calibración.

- Seguimiento inercial: serían los acelerómetros antes mencionados. No precisan sistema emisor/receptor y pueden ser muy compactos. Si se combinan con otros sistemas de seguimiento, como cámaras y puntos de referencia podemos aumentar la fidelidad. Existen dispositivos pequeños e inalámbricos como el MTw Awinda inertial sensors de Xsens Technologies (Enschede, The Netherlands) que pueden analizar el balanceo durante la marcha o en una prueba de Romberg [7], representando una alternativa a la posturografía sobre plataforma.

En el ámbito de la realidad virtual, cuando combinamos el seguimiento de los movimientos de rotación de la cabeza (tres grados de libertad), con los de traslación del propio sujeto en los tres planos del espacio podemos identificar seis grados de libertad. Hasta ahora, para captar estos seis grados, era necesario colocar varias cámaras en el entorno, pero recientemente han surgido dispositivos autónomos en espacios cerrados que, a través del montaje de unas cámaras en el HMD, mapean el medio circundante. Dos ejemplos serían las gafas de realidad virtual HoloLens o las HTC Vive Cosmos.

\section{APLICACIONES DE LA REALIDAD VIRTUAL A LA REHABILITACIÓN VESTIBULAR}

En este apartado destacaremos las oportunidades que estas tecnologías brindan a la rehabilitación vestibular, a través de los diferentes mecanismos de compensación.

EsTimulación OCULOMOTORA. El objetivo es incrementar, a través de la adaptación, la ganancia del reflejo vestíbulo-ocular para estabilizar la mirada. Sin embargo, en ocasiones las pruebas diagnósticas detectan pacientes con un patrón de preferencia visual y ganancias normales. En estos casos la realidad virtual puede ser útil induciendo un conflicto visuo-vestibular, que mejora la dependencia visual por habituación. Los estímulos utilizados pueden ser patrones optoquinéticos o de efecto túnel. Pero también podemos situar al paciente en entornos virtuales similares a los que le provocan mareo en la vida real: un supermercado, una calle con mucha gente... Aunque los estímulos del mundo real suelen ser más potentes [8], en el medio virtual el estímulo es de intensidad controlada y gradual, permitiendo que las actividades tengan una estructura escalonada y que la complejidad de los ejercicios pueda ser progresiva.

Una forma potencial de optimizar la estimulación oculomotora podría ser a través de los sistemas de seguimiento pupilar. Éstos están en continuo desarrollo, y ya existe una implementación para Oculus Rift, un HMD que añade una cámara que monitoriza la dirección de la mirada a 120 fotogramas por segundo, permitiendo optimizar el renderizado de imágenes, especialmente en las zonas donde se centra la visión [9], lo que HTC denomina foveated rendering. Este sistema permitiría aumentar la tolerancia a los escenarios virtuales; pues al quitar detalle a las áreas periféricas de la mirada, disminuiría el mareo generado por la propia realidad virtual, lo que en la literatura anglosajona se denomina virtual reality sickness o 
cybersickness. Incluso asume que sea la dirección de la mirada y no la posición de la cabeza u otro sistema complementario, la que determine la interacción con el entorno.

Se necesitan más trabajos experimentales para valorar si los refinamientos de las gafas de realidad virtual mejorarán los resultados de la rehabilitación vestibular. Ya se han hecho pruebas en sujetos sanos con ejercicios de seguimiento ocular en los que al mismo tiempo se ofrece a la persona información de la dirección de su propia mirada [10].

Finalmente, también tendremos en cuenta la realidad aumentada, un tipo de realidad mixta donde se combinan elementos virtuales en un entorno real. Presenta una mayor complejidad a la hora de poderlo aplicar, pero ya existen gafas que con cristales especiales o recreando el entorno con cámaras, pueden añadir elementos virtuales a lo que vemos. Este sistema podría facilitar la aplicación de terapias de estimulación oculomotora en un medio conocido, como pueda ser nuestra casa o nuestra ciudad.

EsTimulación CEFALOMOTORA. Las gafas de realidad virtual más sencillas, como las basadas en Google Cardboard (Figura 1, derecha) disponen de giroscopios para monitorizar la orientación de la cabeza con tres grados de libertad. Esto podría ser suficiente para la rehabilitación de reflejo cérvicoocular y así suplir la pérdida de ganancia del reflejo vestíbulo-ocular en pacientes con hipofunción vestibular bilateral. Gimmon et al. [7] presentaron un dispositivo de entrenamiento que parece lograr que un paciente acelere la obtención de sacadas agrupadas y encubiertas con los movimientos rápidos de cabeza. Esta unidad combina, en una banda para la cabeza, giroscopios, con punteros láser que se mueven en sentido inverso a los movimientos cefálicos.

Estimulación DEL CONTROL POSTURAL (PLATAFORMAS ESTABILOMÉTRICAS). Las nuevas tecnologías también incorporan plataformas de fuerza para la rehabilitación vestibular. Pueden ser de tipo posturografía estática como la Balance
Rehabilitation Unit (BRU ${ }^{T M}$ ) de Medicaa ${ }^{T M}$ que se combina con unas gafas de realidad virtual que pueden recrear situaciones de la vida real [11] (software $M B S^{T M}$ ). Pero también de posturografía dinámica computarizada como la Bertec's Computerized Dynamic Posturography (CDP) que añade un entorno virtual inmersivo, en vez del entorno visual móvil, con una pantalla en medio, como el modelo de Neurocom. Además, efectúa un estudio de posturografía dinámica monitorizando cada pie por separado. Sería similar al representado en la derecha de la Figura 1, aunque el Bertec no ofrece imágenes tridimensionales sino una pantalla de grandes dimensiones. Con un precio actual muy elevado, es uno de los dispositivos que se postula como el futuro gold standard de la posturografía dinámica combinada con un entorno virtual [12].

La Wii Balance Board (WBB) de Nintendo es una plataforma de fuerza de la que se han realizado varios estudios que la validan como plataforma de posturografía estática $[13,14]$, reafirmándose el concepto de que para una buena rehabilitación vestibular no se precisan dispositivos costosos. Incluso se ha creado una plataforma web SeeSway [15] que cruza y analiza datos de posturografía obtenidos desde diferentes plataformas o sistemas de captura de movimiento.

Existen otras formas de analizar el control postural, como los medios de seguimiento ocular que obtienen la posición de un sujeto respecto al contexto. Un ejemplo es la Microsoft Kinnect ${ }^{T M}$ que, con dos cámaras, una de ellas infrarroja, crea un mapa de profundidad tras la emisión de un patrón de haces de luz infrarroja. En un estudio de 2012 [16], este dispositivo mostró una capacidad similar a un sistema de captura de movimiento con múltiples cámaras, incluso como herramienta para analizar el control postural.

Sustitución vestibular. Aunque algunos autores prefieren hablar de mecanismos de adicción sensorial, mediante la retroalimentación es posible ayudar a un sistema vestibular deficitario [17]. Destacamos las siguientes posibilidades: 
- Si el paciente oye relativamente bien, la ayuda auditiva añadida a la rehabilitación vestibular parece mejorar el equilibrio [18].

- También disponemos de sistemas de estimulación vibrotactil como Vertiguard ${ }^{\circ}$ [19] o Biodex Vibrotactile $e^{\mathrm{Tm}}$ System, que gracias a sus giroscopios pueden detectar cambios en la postura y enviar estímulos vibratorios a diferentes partes del cuerpo, para optimizar las estrategias de control postural. Otro aparato similar sería el Sway Star ${ }^{T M}$ que monitoriza la inclinación del cuerpo y que puede combinarse con una cinta para la cabeza BalanceFreedom ${ }^{T M}$ que da pistas auditivas, vibrotactiles y visuales sobre el balanceo.

- Otro dispositivo de sustitución es el BrainPort Balance Plus de Wicab, que mediante estimulación lingual electrotactil, informa de los cambios de posición del cuerpo. Su empleo en rehabilitación vestibular parece que podría acelerar la recuperación [20].

- Y por último los implantes vestibulares, que representan el futuro para la restitución del sistema vestibular y aunque prometedores, están aún en fase experimental hasta que se logren calibrar sin interferir con la función vestibular residual [21].

\section{DISCUSIÓN}

Aunque no existen muchos artículos científicos que analicen la eficacia de la realidad virtual en la rehabilitación vestibular, la mayor parte concluyen que este tipo de tratamientos son seguros, útiles para mejorar el equilibrio y valorados positivamente por los pacientes gracias a su aspecto lúdico.

Sin embargo, todavía surgen muchas dudas acerca de sus indicaciones y efectos adversos. La realidad virtual puede provocar síntomas similares a la cinestosis y cambios oculomotores transitorios. También puede inducir una desviación postural y un incremento en la oscilación corporal. [22] La prevalencia de estos efectos no deseados está poco documentada en muchos estudios. Los futuros ensayos sobre la terapia con realidad virtual deberían recoger estos efectos [23]. Existen incluso herramientas para su medición como la Simulator Sickness Questionnaire o incluso, una adaptación más actual, Virtual reality sickness questionnaire [24].

La Tesis Doctoral de Alahmari en 2012, trata sobre la seguridad y validez de una unidad de rehabilitación, combinada con un entorno virtual cúbico tipo CAVE. En la misma, también desarrolla un ensayo con dos grupos de 20 pacientes administrándoles a unos una terapia física personalizada y a los otros una terapia basada en realidad virtual y no encuentra diferencias significativas entre ambos [25].

Bergeron et al. [23] presentaron en 2015 un metanálisis donde todos los estudios incluidos indicaban que la rehabilitación apoyada con realidad virtual tiene un efecto positivo y es bien tolerado. El principal criterio de éxito para mejorar los síntomas es la duración del entrenamiento, pero la complejidad del aparataje no supone una mayor eficiencia en los resultados.

En un ensayo controlado y aleatorizado de 2015, en el que participaron 71 pacientes con vestibulopatía unilateral, se observó que los ejercicios sobre una plataforma tipo WBB complementados con videojuegos, no obtuvieron un resultado mejor a los 6 meses que los realizados sobre gomaespuma, aunque los pacientes se cansaron menos y disfrutaron más [26].

Hsu et al. (2016), plantearon un estudio piloto con 70 pacientes, 34 controles y 36 con inestabilidad crónica causada por enfermedad de Ménière, en donde todos recibieron terapia con realidad virtual. Los pacientes tratados mejoraron su control direccional y su balanceo, especialmente en las primeras fases de la enfermedad [27].

Viziano et al. (2018), estudiaron los efectos a largo plazo de la rehabilitación vestibular con HMD. De los 47 pacientes con hipofunción vestibular unilateral, a 24 se le administró una terapia vestibular convencional y a 23 con gafas de realidad virtual durante un mes. Este último grupo mejoró significativamente al año, tanto en la ganancia como en los parámetros posturográficos [28]. 
A la vista de los resultados publicados parece lógico expresar, que se hace necesario estandarizar los protocolos de evaluación y tratamiento, documentando en futuros estudios, los efectos secundarios de la rehabilitación con realidad virtual.

\section{CONCLUSIONES}

Las nuevas tecnologías aplicadas a la rehabilitación vestibular además de recalibrar los sistemas que integran el equilibrio, siguiendo los paradigmas clásicos de compensación, nos ayudan a profundizar en el análisis de los desajustes o secuelas derivados de una vestibulopatía.

La variedad tecnológica y su diferente complejidad permite diversificar su uso. Los dispositivos más sencillos, aprovechando incluso el teléfono móvil, puede ser empleados en el domicilio. Otros intermedios, pero que precisan de una cierta inversión y supervisión, serían accesibles en las unidades de otoneurología. Y finalmente, los sistemas más sofisticados estarían orientados a la investigación.

En cualquiera de los casos, se trata de una fisioterapia más lúdica, que facilita la adherencia al tratamiento e incorpora su propio sistema de evaluación. Se necesitan más ensayos clínicos que analicen la eficacia terapéutica de estas tecnologías en relación con la terapia física tradicional y una mayor colaboración entre ingenieros, clínicos e investigadores para optimizar estos nuevos instrumentos.

\section{AGRADECIMIENTOS}

Mi más sincero agradecimiento al Dr. José Ignacio Benito Orejas por guiarme en la realización de este trabajo.

\section{BIBLIOGRAFÍA}

1. Garrett B, Taverner T, Gromala D, Tao G, Cordingley E, Sun C. Virtual Reality Clinical Research: Promises and Challenges. JMIR Serious Games. 2017;6(4):e10839.
2. Schulze, J. How Virtual Reality Works? [curso online] edX: UCSanDiegoX: CSE165x; 2017. Disponible en: https://courses.edx.org/courses/ course-v1:UCSanDiegoX+CSE165x+2T2017/ course/. [Citado el 7-8-2019].

3. Brooks F. What's real about virtual reality? IEEE Computer Graphics and Applications, 1999;19(6):16-27.

4. Riva G, Wiederhold BK, Mantovani F. Neuroscience of Virtual Reality: From Virtual Exposure to Embodied Medicine. Cyberpsychol Behav Soc Netw. 2019;22(1):82-96.

5. Saeed A, Foaud L, Fattouh L. Environments and System Types of Virtual Reality Technology in STEM: a Survey. International Journal of Advanced Computer Science and Applications. 2017;8(6).

6. Febretti A, Nishimoto A, Thigpen T, Talandis J, Long L, Pirtle J et al. CAVE2: a hybrid reality environment for immersive simulation and information analysis. Proceedings. SPIEVL 8649, The Engineering Reality of Virtual Reality. 2013;864903. Disponible en: https://www.evl.uic.edu/documents/ spie13paper-final-2.pdf. [Citado el 7-8-2019].

7. Gimmon Y, Migliaccio A, Kim K, Schubert M. VOR adaptation training and retention in a patient with profound bilateral vestibular hypofunction. Laryngoscope. 2019; Feb 19 [publicación electrónica antes de impresión]. https://onlinelibrary. wiley.com/doi/pdf/10.1002/lary.27838. [Citado el 7-8-2019].

8. Hain T. Virtual reality for Vestibular rehabilitation treatment. Dizziness-and-balance.com. [Internet]. Chicago, IL; [Fecha de actualización: 13 de febrero de 2016; fecha de consulta: 1 de Julio de 2019]. Disponible en: https://www.dizziness-and-balance. com/treatment/rehab/virtual_reality.html. [Citado el 7-8-2019].

9. Pohl D, Zhang X, Bulling A, Combining eye tracking with optimizations for lens astigmatism in modern wide-angle HMDs. 2016 IEEE Virtual Reality (VR); 2016 Marzo 19-23; Greenville, SC: IEEE publisher;c 2016. p. 269-70.

10. Park J, Jeon H, Lim E, Koo J, Lee H, Kim H et al. Feasibility of Eye Tracking Assisted Vestibular Rehabilitation Strategy Using Immersive Virtual Reality. Clin Exp Otorhinolaryngol. 2019. Dispo- 
nible en: https://www.e-ceo.org/journal/view. php?doi=10.21053/ceo.2018.01592. [Citado el 7-8-2019].

11. Yamamoto MEI, Ganança CF. Posturografia com estímulos de realidade virtual nas diferentes disfunções vestibulares. Rev. Soc. Bras. Fonoaudiol. 2012;17(1):54-60.

12. Trueblood P, Rivera M, Lopez C, Bentley C, Wubenhorst N. Age-based normative data for a computerized dynamic posturography system that uses a virtual visual surround environment. Acta Otolaryngol. 2018;138(7):597-602.

13. Domènech-Vadillo E, Aguilera-Aguilera G, Sánchez-Blanco C, Batuecas-Caletrio A, Guajardo C, Pérez $\mathrm{N}$ et al. Normative data for static balance testing in healthy individuals using open source computerized posturography. Eur Arch Otorhinolaryngol. 2019 Jan;276(1):41-8.

14. Albiol-Pérez S, Gil-Gómez JA, Alcañiz M, Llorens $\mathrm{R}$, Colomer C.Use of the Wii balance board system in vestibular rehabilitation. En: INTERACCION ' 12 Proceedings of the 13th International Conference on Interacción Persona-Ordenador. Article No. 11. Elche, Spain - October 03 - 05, 2012. ACM, editores. Nueva York;2012. Articulo n ${ }^{\circ} 11$. p.1-4. doi: 10.1145/2379636.2379647.

15. Clark R, Pua Y. SeeSway - A free web-based system for analysing and exploring standing balance data. Comput Methods Programs Biomed. 2018;159:31-6.

16. Clark R, Pua Y, Fortin K, Ritchie C, Webster K, Denehy L et al. Validity of the Microsoft Kinect for assessment of postural control. Gait Posture. 2012;36(3):372-7.

17. Sienko K, Seidler R, Carender W, Goodworth A, Whitney S, Peterka R. Potential Mechanisms of Sensory Augmentation Systems on Human Balance Control. Front. Neurol. 2018;9:944.

18. Dozza M, Chiari L, Horak F. Audio-Biofeedback Improves Balance in Patients With Bilateral Vestibular Loss. Arch Phys Med Rehabil. 2005;86(7):1401-03.

19. Brugnera C, Bittar RSM, Greters ME, Basta D. Effects of vibrotactile vestibular substitution on vestibular rehabilitation - preliminary study. Braz J Otorhinolaryngol. 2015;81:616-21.

20. Danilov Y, Tyler M, Skinner K, Hogle R, Bach-YRita P. Efficacy of electrotactile vestibular subs- titution in patients with peripheral and central vestibular loss. J Vesti Res. 2007;17:119-30.

21. van de Berg R, Guinand N, Ranieri M, Cavuscens S, Khoa Nguyen T, Guyot J et al. The Vestibular Implant Input Interacts with Residual Natural Function. Front Neurol 2017;8:644.

22. Herdman., S. and Clendaniel, R. (2014). Vestibular Rehabilitation. 4th ed. Philadelphia: F.A. Davis Company, p.539.

23. Bergeron M, Lortie C, Guitton M. Use of Virtual Reality Tools for Vestibular Disorders Rehabilitation: A Comprehensive Analysis. Adv Med. 2015;2015:1-9.

24. Kim HK, Park J, Choi Y, Choe M. Virtual reality sickness questionnaire (VRSQ): Motion sickness measurement index in a virtual reality environment. Appl Ergon. 2018 May;69:66-73.

25. Alahmari, K. The Use of Technology in Vestibular Rehabilitation and Balance Assessment. [Tesis en internet]. [Pittsburgh]: University of Pittsburgh; 2012222 p. Disponible en: http://d-scholarship. pitt.edu/11679/. [Citado el 7-8-2019].

26. Meldrum D, Herdman S, Vance R, Murray D, Malone K, Duffy D et al. Effectiveness of Conventional Versus Virtual Reality-Based Balance Exercises in Vestibular Rehabilitation for Unilateral Peripheral Vestibular Loss: Results of a Randomized Controlled Trial. Arch Phys Med Rehabil. 2015;96(7):1319-28.e1.

27. Hsu S, Fang T, Yeh S, Su M, Wang P, Wang V. Three-dimensional, virtual reality vestibular rehabilitation for chronic imbalance problem caused by Ménière's disease: a pilot study. Disabil Rehabil. 2016;39(16):1601-6.

28. Viziano A, Micarelli A, Augimeri I, Micarelli D, Alessandrini M (2018) Long-term effects of vestibular rehabilitation and head-mounted gaming task procedure in unilateral vestibular hypofunction: a 12-month follow-up of a randomized controlled trial. Clin Rehabil. 2019;33(1):24-33.

\section{ENLACES RELACIONADOS}

Seeway. Online balance data processing. Disponible en: http://www.rehabtools.org/seesway.html. [Citado 23-7-2019]. 